\title{
EFECTOS DEL CONTROL DEL PICUDO NEGRO (Cosmopolites sordidus) EN EL PLÁTANO ${ }^{1}$
}

\author{
Ignacio Armendáriz², Pablo Anibal Landázuri , José Marco Taco ${ }^{3}$, Santiago M. Ulloa ${ }^{3}$
}

\section{RESUMEN}

Efectos del control del picudo negro (Cosmopolites sordidus) en el plátano. El objetivo del estudio fue comprobar el efecto en el plátano de las capturas masivas de adultos con feromonas y de la aplicación de hongos entomopatógenos sobre Cosmopolites sordidus. La investigación se realizó en el Carmen, Manabí, Ecuador, de diciembre 2013 a octubre 2014 en una parcela de nueva plantación de plátano Barraganete y se valoró el efecto de la eliminación de restos vegetales de las parcelas. El diseño experimental fue al azar con tres repeticiones. En laboratorio se comprobó la infectividad de la cepa de Beauveria bassiana sobre adultos del picudo negro y del picudo café (Metamasius hemipterus) (92,80 y 100\% en $M$. hemipterus frente a 46,70 y $16,20 \%$ en $C$. sordidus). Las capturas de adultos fueron continuas a lo largo del ensayo, con una media de 0,37 adultos/trampa/día. Los datos agronómicos indicaron una diferencia positiva y significativa (análisis de la varianza) de los tratamientos frente al control en el perímetro del pseudotallo ( $F$ de 4,982) y en el peso de los racimos entre las parcelas limpias con feromonas y con hongos limpias frente al control $(\mathrm{F}=3,994)$. Al comparar los tratamientos el peso del racimo y la longitud de los dedos fueron significativamente mayores en las parcelas de feromonas frente a las de hongos $(\mathrm{F}=4,508)$. Las capturas de adultos con feromonas no estuvieron condicionadas por la limpieza de las parcelas, aunque esta sí incidió en la altura de las plantas $(\mathrm{F}=4,245)$.

Palabras clave: Beauveria bassiana, Cosmopolites sordidus, captura masiva, Musa sp.

\section{ABSTRACT}

Effects of black weevil control (Cosmopolites sordidus) in plantains. The aim of this study was to test the effect of mass capture of adults using pheromone traps and applying entomopathogenic fungi on Cosmopolites sordidus. The research was conducted in El Carmen, Manabí, Ecuador, from December 2013 to October 2014, in a plot of new Barraganete plantain plantation. The effect of plant remains removal from the plots was also assessed. The experimental design was randomized complete block with three repetitions. The infectivity of Beauveria bassiana on adults of black weevil and brown weevil (Metamasius hemipterus) was evaluated in the laboratory(92.80 and 100\% in $M$. hemipterus versus 46.70 and $16.20 \%$ in C. sordidus). Adult catches were continuous throughout the experiment, with an average of 0.37 adults/trap/day. Agronomic data indicated a positive and significant (analysis of variance) difference of treatment versus control at the perimeter of the pseudostem $(4.982 \mathrm{~F} *)$ and bunch weight between plots with pheromones and plots with clean fungi versus control $(\mathrm{F}=3.994 * *)$. When comparing treatments, bunch weight and finger length were significantly higher in the plots treated with pheromones compared to the ones treated with fungi $\left(\mathrm{F}=4.508^{* *}\right)$. Catches of adults with pheromones were not conditioned by the cleaning of plots, though this did affect the plant height $(\mathrm{F}=4.245 *)$.

Keywords: Beauveria bassiana, Cosmopolites sordidus, massive capture, Musa sp.

\footnotetext{
1 Recibido: 27 de julio, 2015. Aceptado: 19 de octubre, 2015. Este trabajo forma parte del proyecto de investigación "Control integrado del picudo negro del banano, Cosmopolites sordidus, en Ecuador" financiado por la SENESCYT.

2 Universidad Tecnológica Equinoccial, Facultad de Medicina Eugenio Espejo, Escuela de Odontología. Avda Mariana de Jesús y Mariscal Sucre, Quito, Ecuador. i.armendariz2009@gmail.com (autor para correspondencia).

3 Universidad de las Fuerzas Armadas, ESPE. Av. General Rumiñahui s.n. Sangolquí. Ecuador. P.O.Box 171-5-231B. IASA. palandazuri@ espe.edu.ec,jmtaco@espe.edu.ec, smulloa@espe.edu.ec
} 


\section{INTRODUCCIÓN}

El plátano (Musa AAB) en Ecuador es considerado un cultivo de creciente importancia socioeconómica, pues este país ocupa el cuarto lugar como productor de esta fruta a nivel mundial, con una producción anual de 7931000 toneladas (Fundación PRODUCE de Guerrero, A.C., 2012).

Las principales variedades empleadas son Dominico y Barraganete, con una exportación de 90 000 t. Para el año 2011 se reportaron en el país un total de 144981 ha de plátano, de las cuales 86712 estaban bajo el sistema de monocultivo y 58269 se encontraban asociadas con otros cultivos (INEC, 2011). Destacan las provincias de El Oro, Esmeraldas, Guayas, Loja, Los Ríos y Manabí.

El picudo negro del plátano, Cosmopolites sordidus (Germar) (Coleoptera: Dryophthoridae), es la plaga principal en los cultivos de plátano y banano (Gold et al., 2005). Al igual que las musáceas, este insecto es originario del sureste de Asia, donde no representa un problema crítico debido a la presencia de controladores naturales (Abera-Kalibata et al., 2006).

El control de esta plaga en plantaciones comerciales depende del uso de insecticidas (principalmente organofosforados, carbamatos y piretroides), con resultados no siempre satisfactorios (Gold et al., 2001). La fumigación con insecticidas puede afectar la salud de los aplicadores y de los consumidores, asî como al ambiente (Muñoz-Ruiz, 2007). Por otra parte, el menor costo de los métodos de manejo integrado de plagas y su efectividad animan a muchos productores a su empleo (Aguilar et al., 2014). Entre ellos están el uso de hongos entomopatógenos y la captura masiva de adultos del insecto mediante trampas de feromonas (Gold y Messiaen, 2000). El control de las poblaciones del picudo mediante su captura con trampas de feromonas o con sustancias alimenticias (De Graaf et al, 2005; Rhino et al., 2010), es parte habitual de una estrategia de reducir su umbral de daño, ya que los adultos capturados son eliminados de la parcela. Lopes et al. (2014) proponen una combinación del uso de feromonas y hongos en una estrategia de atraer y matar. Los adultos atraídos por la feromona son potencialmente infectados por el hongo y vuelven a la parcela, donde podrán infectar a otros adultos, dado el carácter asociativo de la especie (De Graaf et al., 2005). Son varios los trabajos realizados que demuestran la efectividad en el control de los hongos entomopatógenos, especialmente cepas de Beauveria bassiana y Metarhizium anisopliae (Gold y Messiaen, 2000; Akello et al., 2008; Fancelli et al., 2013).

La pérdida de producción causada por el picudo puede llegar a un $42 \%$ de la cosecha, debido al deterioro de la plantación por volcamiento de las plantas en la temporada húmeda (Gold et al., 2004). El picudo provoca un daño directo, producido por las larvas al alimentarse del cormo, que causa la reducción de la producción y la vida útil de la plantación (Gold et al., 2005). Los ataques de los picudos negros interfieren con la iniciación de las raíces, matan las raíces existentes, limitan la absorción de nutrientes, reducen el vigor de las plantas, demoran la floración y aumentan la susceptibilidad a plagas y enfermedades (Gold y Messiaen, 2000). Los daños en producción se relacionan con la pérdida de plantas por muerte o volcamiento, especialmente en épocas de lluvia, fallas en la fructificación y reducción del peso del racimo. El volcamiento de plantas puede originar igualmente huecos en las plantaciones y exigir replantaciones, que van a descompensar el manejo de la parcela, al tener plantas en distinto estado de crecimiento.

El crecimiento de las poblaciones del picudo negro es lento, y su ataque puede interferir en el establecimiento de parcelas y en el acortamiento de su vida útil (Gold et al., 2005). La especie presenta un comportamiento críptico, ya que los adultos tienen una actividad nocturna, permaneciendo ocultos de día en la vegetación. Además, las larvas actúan en el cormo, por lo cual únicamente si se corta el pseudotallo y se explora el cormo son visibles las galerías. Más evidente es otra especie de picudo, el rayado o café (Metamasius hemipterus Olivier, 1807), cuyas larvas se alimentan principalmente de material vegetal cortado y cuyos adultos acuden a los cortes de racimos cosechados. Sin embargo, la presencia de esta especie no suele ocasionar daños, salvo en plataneras enfermas y debilitadas (Jiménez et al., 2012).

Los picudos son atraídos por los compuestos volátiles liberados por plantas dañadas y cortadas. Los desplazamientos de los adultos son por lo general muy cortos, con estimaciones de $50 \mathrm{~m}$ en tres meses (Gold et al., 2005), por lo que el daño en una platanera nueva aparece en los sectores que se van extendiendo. Los picudos raramente vuelan, la diseminación ocurre principalmente a través del material de plantación infestado. 
Bajo condiciones tropicales, el tiempo que le toma a un huevo convertirse en un adulto es de ocho semanas (Gold y Messiaen, 2000), aunque puede alargarse varias semanas según las condiciones climáticas, la variedad, edad y estado de la planta. El desarrollo de los huevos no ocurre con temperaturas menores de $12{ }^{\circ} \mathrm{C}$ (Traore et al., 1993), umbral que limita el movimiento de los adultos. Dadas las condiciones climáticas de las zonas productoras de musáceas en Ecuador se puede esperar un número elevado de generaciones de picudo y su actividad durante todo el año (Gold et al., 2001).

La cantidad de picudos que se encuentran en plantaciones nuevas es escasa, con bajas tasas de oviposición, y crecimiento de población lento; el problema se manifiesta con mayor frecuencia en el segundo ciclo del cultivo. Las pérdidas del rendimiento en el cultivo pueden ir de un 5\% en el primer ciclo a más de $40 \%$ en el tercero. En áreas donde los bananos o plátanos se renuevan después de uno a tres años, las poblaciones de picudo negro pueden no tener suficiente tiempo para crecer hasta niveles de plaga (Gold y Messiaen, 2000).

Las prácticas culturales son un conjunto de operaciones que están orientadas a mejorar la producción de un cultivo y que en ocasiones colaboran con el control de plagas y enfermedades. En principio plantas más vigorosas van a ser más resistentes al ataque del picudo (INIBAP, 1998). Los adultos del picudo son atraídos por la humedad y los fermentos derivados de la descomposición de los residuos de cosechas, como cormos y tallos (Quijije, 2003). Es de gran importancia eliminar dichos residuos, ya que sirven de atrayente y refugio para estos insectos. Se señala que el control del picudo negro es principalmente de tipo cultural; se debe mantener el aspecto de la plantación libre de malezas, con fertilizaciones adecuadas y riegos oportunos, realizar muestreos frecuentemente y destruir las plantas que se encuentran afectadas. La limpieza y extracción de los restos de material ayuda al control al eliminar refugios, zonas de alimentación y atracción. Pero este esfuerzo presenta el inconveniente de que esa materia no acabe asimilándose al suelo tras su descomposición. Ante esto se presentan dos alternativas; la primera es la adición de microorganismos descomponedores de materia orgánica para acelerar el proceso. La segunda es el traslado a un lugar alejado y realizar su compostaje, para posteriormente añadir el compost a la plantación, p.ej. en el momento de la renovación. Masanza et al. (2005) proponen como solución el corte en pequeños trozos de las plantas cosechadas para evitar en lo posible el desarrollo de las larvas, aunque en este material se desarrollan las larvas del picudo café, limitándose las del picudo negro al cormo.

Por la fuerte afectación del picudo a las plantaciones, la ausencia general de control del mismo, con el apoyo del Programa PROMETEO de la SENESCYT, se han realizado distintas investigaciones para el control del picudo.

El objetivo del estudio fue comprobar el efecto en el plátano de las capturas masivas de adultos con feromonas y de la aplicación de hongos entomopatógenos sobre Cosmopolites sordidus.

\section{MATERIALES Y MÉTODOS}

Se seleccionó una parcela de plátano Barraganete en la localidad de El Carmen, Manabí, Ecuador. La misma fue plantada el 28 de agosto de 2013 y replantada el 8 de noviembre, con una distancia de siembra de $3 \mathrm{x}$ $2,6 \mathrm{~m}$, sobre un cultivo anterior de cacao. Las plantas procedían de una plantación cercana y les fue aplicado carbofurano en plantación ( $5 \mathrm{~g} /$ planta). El clima de la zona es tropical megatérmico húmedo y los suelos pertenecen al tipo inceptisol (MAGAP, 2015).

El diseño empleado fue al azar y con tres repeticiones (Figura 1), para evitar el sesgo de las

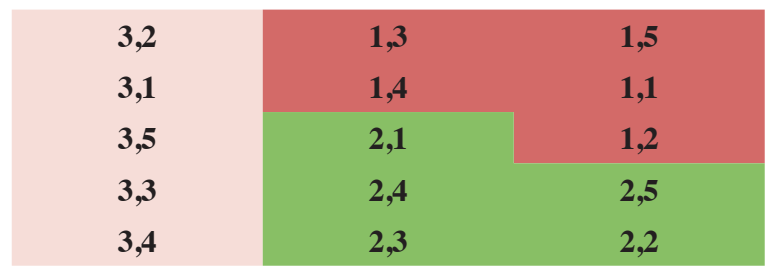

Figura 1. Distribución de los tratamientos para el control del picudo negro del plátano. Los números hacen referencia el primero a las repeticiones $\left(1^{\mathrm{a}}, 2^{\mathrm{a}}\right.$ o $\left.3^{\mathrm{a}}\right)$ y el segundo al tratamiento (1. hongos, limpio; 2. hongos, sucio; 3 . feromonas, limpio; 4. feromonas, sucio; 5. control). Manabí, Ecuador. 2013-2014.

Figure 1. Distribution of treatments to control the black weevil in plantains. The numbers refer, the first one to the repetitions $\left(1^{\text {st }}, 2^{\text {nd }}\right.$ or $\left.3^{\text {rd }}\right)$ and the second one to the treatment (1. clean, mushrooms; 2. dirty, mushrooms; 3. clean, pheromones; 4. dirty, pheromones; 5. control). Manabí, Ecuador. 2013-2014. 
distintas condiciones de la parcela, como la pendiente o la profundidad del suelo. En total se establecieron cinco filas y tres columnas (Figura 1). Se aplicaron los siguientes tratamientos:

1. Discos de plátano con hongos y limpieza (eliminación de restos vegetales superficiales tanto del cultivo anterior de cacao como los producidos en las labores culturales de deshoje, eliminación de brotes y cosecha).

2. Discos con hongos, sin limpieza.

3. Trampas de feromona con limpieza.

4. Trampas de feromona sin limpieza.

5. Control, sin capturas ni limpieza.

Cada subparcela constó de dieciocho plantas, separadas por una calle, con dimensiones de subparcela de $6 \times 9 \mathrm{~m}$. El tratamiento de hongos o feromonas fue aplicado en el centro de la subparcela. Entre cada punto de tratamiento existía una distancia de $11 \mathrm{~m}$ entre filas y 20 m entre columnas. Los datos agronómicos fueron obtenidos de ocho plantas centrales más una de borde.

El 15 de noviembre se realizó la limpieza de las subparcelas para retirar los restos del cultivo anterior de cacao. El objetivo de esta diferenciación fue compobar si los restos vegetales en la subparcela influyen en la captura de adultos con feromonas. La feromona comercial, la sordidina, es análoga a un derivado de las sustancias de descomposición de los pseudotallos (De Graaf et al., 2005). La presencia de restos vegetales de plátano, provenientes de las técnicas culturales (deshojes, tallos cortados tras la cosecha), puede interferir en la correcta difusión de la feromona, así como servir de lugares de refugio diurno a los adultos. Como técnicas culturales se realizaron dos aplicaciones de clorotalonil para el combate de Sigatoka (720 g/ha), y dos de quizalofop-p-etil como herbicida (720 g/ha), deshierbes manuales, deshojes y abonados.

El 29 de noviembre se colocaron seis trampas de feromonas, cuatro en recipientes amarillos de dos piezas que encajaban dejando una abertura central para la entrada de los picudos, y dos en contenedores de plástico blanco, por falta de disponibilidad de los otros recipientes $(12 \mathrm{~cm}$ de diámetro superior y $10 \mathrm{~cm}$ de altura), de los usados habitualmente para el transporte de comida. La posición de los recipientes se alternó durante el estudio para evitar el sesgo de capturas asociado al tipo de trampa, ya que en los recipientes sin tapa es posible que algunos adultos se escapen. Las trampas de colocaron en el centro de cada subparcela, enterradas a ras de tierra hasta su abertura y se llenaron con agua para evitar la fuga de los adultos. Los saquetes de feromonas fueron cambiados cada tres meses, según las instrucciones de los fabricantes (ScyllAgro, 2015). Las trampas fueron revisadas mayoritariamente con un intervalo semanal. Con las capturas anotadas se realizó el cálculo de adulto/trampa/día, diviendo la media de las seis trampas de captura por los días transcurridos entre las anotaciones. Ello permitió homogeneizar la representación y soslayar el problema de intervalos de tiempo diferentes en la revisión de las trampas.

El hongo entomopatógeno utilizado fue una cepa de Beauveria bassiana esporulada en arroz, aislada en el Centro de Ingeniería en Ciencias Agropecuarias (IASA 1) de la Universidad de las Fuerzas Armadas ESPE. Se realizaron aplicaciones quincenales en campo en pseudotallos de plátano recién cortados, de unos 30 $\mathrm{cm}$ de longitud y 20 a 30 de diámetro, iniciando los primeros días de enero de 2014. En cada pseudotallo cortado se aplicó en su interior unos $40 \mathrm{~g}$ de arroz esporulado, en los que visualmente se comprobara un buen desarrollo del micelio. Entre los meses de marzo a julio se midió la altura de la planta $(\mathrm{cm})$ y el perímetro del pseudotallo $(\mathrm{cm})$ a $30 \mathrm{~cm}$ en nueve plantas por subparcela (135 en total), con una cinta métrica. En el momento de la cosecha se tomaron otros parámetros de variables productivas, como el peso del racimo $(\mathrm{kg})$ y longitud de dedos $(\mathrm{cm})$, para cinco plantas por subparcela, en total 75 plantas. En cada subparcela cuatro plantas cosechadas fueron cortadas a ras de tierra, a nivel del cormo, para anotar las galerías de larvas del picudo. Igualmente se llevaron a cabo ensayos de patogenicidad de la cepa de $B$. bassiana sobre adultos de ambas especies de picudo capturados en campo. En laboratorio, se depositaron en cajas petri y se les aplicaron $2,5 \mathrm{ml}$ de una suspensión preparada con el lavado de una placa petri de colonia esporulada con $5 \mathrm{ml}$ de agua destilada. Los insectos permanecieron en las cajas durante dos horas. Posteriormente, se pasaron a botes de plástico con pseudotallo de plátano para su alimentación en condiciones de laboratorio (aproximadamente $20^{\circ} \mathrm{C}$ y 12/12 horas luz/oscuridad). A los catorce días se comprobó la presencia o ausencia de micelio en los animales muertos.

Los análisis estadísticos se realizaron con el programa $\mathrm{R}$ agricolae y consistieron en análisis de varianza y pruebas de comparación de medias de tratamientos. Se realizaron previamente pruebas de normalidad (test de Shapiro y Wilks, 1965), comprobando que todos los datos cumplían las condiciones. 


\section{RESULTADOS Y DISCUSIÓN}

Las capturas de picudos fueron elevadas al comienzo del ensayo, con un total de 1,80 adultos/ trampa/día. En el mes de julio hubo un segundo pico con valores de 1,10 adultos/trampa/día. Para el total del ensayo la media de capturas fue de 0,37 adultos/ trampa/día (Figura 2). Con la variante de limpieza de las subparcelas se pretendió establecer la relación entre la disminución de lugares de refugio para los adultos y la menor interferencia del material vegetal con la acción de las feromonas. Sin embargo, no hubo diferencias estadísticas en las capturas de adultos entre subparcelas limpias y sucias, respectivamente $(\mathrm{F}=0,516)$.

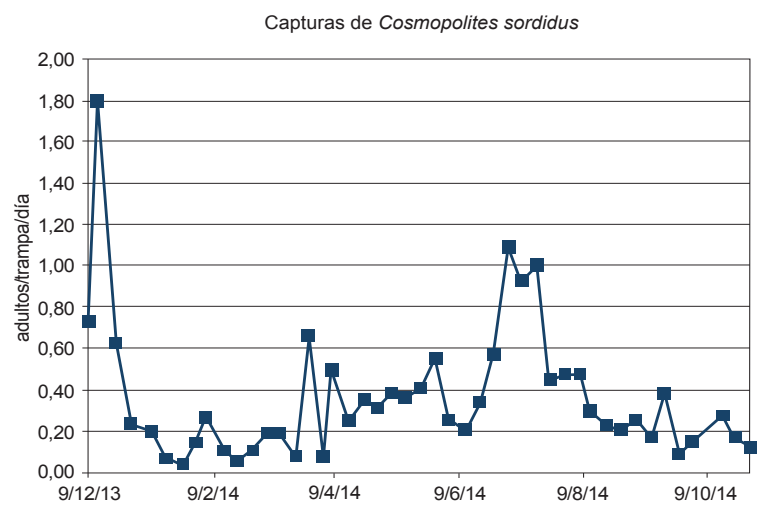

Figura 2. Captura de adultos de Cosmopolites sordidus en trampas de feromonas por trampa y día en una parcela de plátano Barraganete. El Carmen, Manabí, Ecuador. Diciembre 2013 a octubre de 2014.

Figure 2. Capture of Cosmopolites sordidus adults in pheromone traps per traps and per day in a plot of Barraganete plantain. El Carmen, Manabí, Ecuador. December 2013 to October 2014.

La altura de las plantas fue significativamente superior en las subparcelas limpias frente a las sucias $(\mathrm{F}=4,245)$. El crecimiento fue continuo en las plantas entre las semanas 28 y 44 , momento en el que empieza la floración y se detiene el crecimiento (Figura 3). Respecto al perímetro del pseudotallo a 30 $\mathrm{cm}$ (Figura 4) en las subparcelas en que se aplicaron hongos, fue superior en las sucias; mientras que en el tratamiento con feromonas el perímetro fue superior
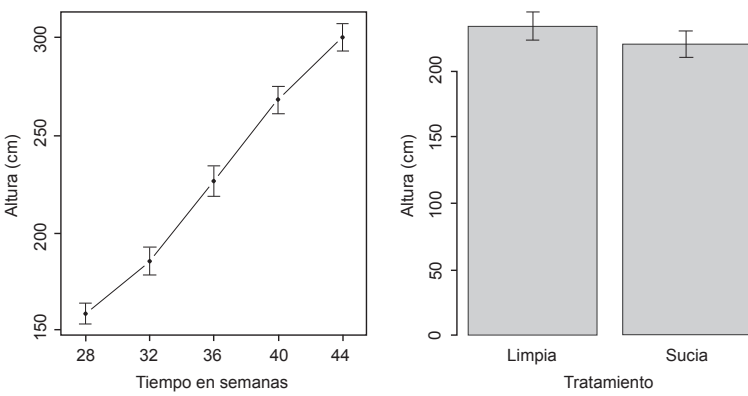

Figura 3. Altura de las plantas de plátano Barraganete hasta el primer par de hojas (media de las 135 plantas en la gráfica izquierda y medias de 54 plantas en la de la derecha). El Carmen, Manabí, Ecuador. Marzo a julio de 2014 .

Figure 3. Height of the Barraganete plantain plant to the first pair of leaves (average of 135 plants in the left figure and average of 54 plants in the right one). El Carmen, Manabí, Ecuador. March to July 2014.

en las subparcelas limpias. En estas comparaciones únicamente fue significativa la comparación entre subparcelas limpias y el control, con una F de 4,982. Igualmente el perímetro del pseudotallo se incrementó entre las semanas 28 y 44 (Figura 4).

El peso de los racimos (Figura 5) se vio afectado por los tratamientos de forma significativa $(F=3,994)$; en los
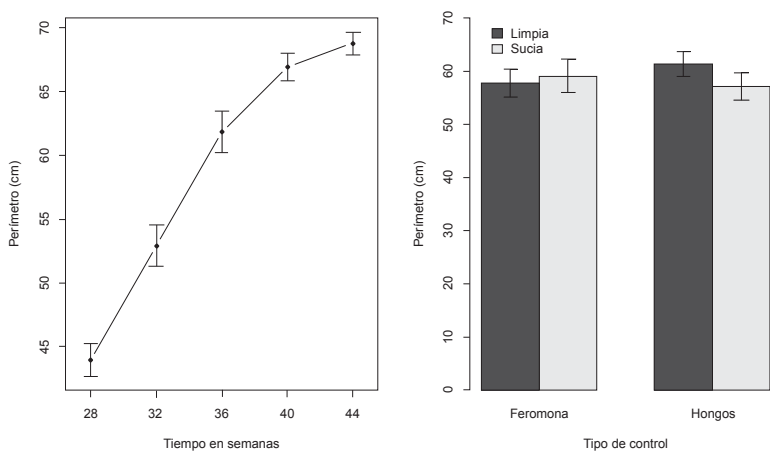

Figura 4. Perímetro de las plantas de plátano Barraganete a $30 \mathrm{~cm}$ (media de las 135 plantas en la gráfica izquierda y medias de 27 plantas en la de la derecha). El Carmen, Manabí, Ecuador. Marzo a julio de 2014.

Figure 4. Perimeter of Barraganete plantain plants at 30 $\mathrm{cm}$ (average of 135 plants in the left figure and average of 27 plants in the right one). El Carmen, Manabí, Ecuador. March to July 2014. 


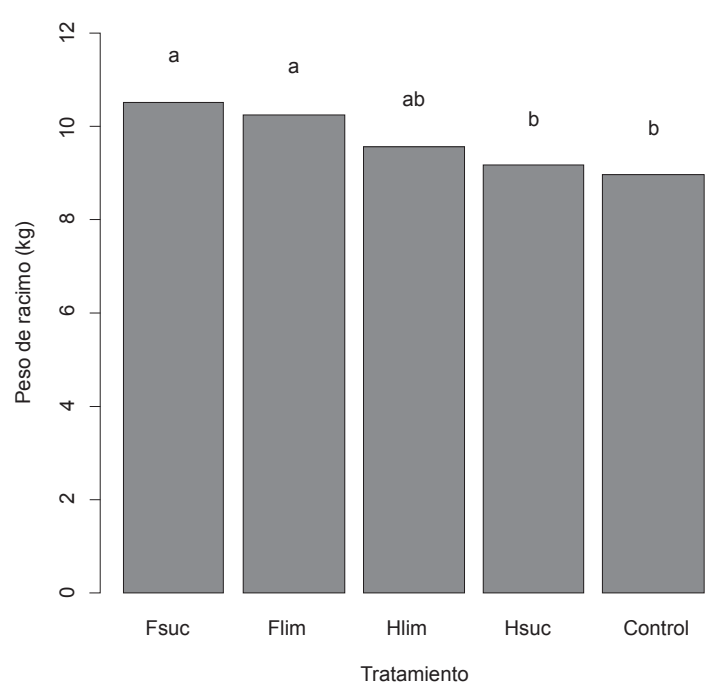

Figura 5. Peso de los racimos de plátano Barraganete en kg por tratamientos. El Carmen, Manabí, Ecuador. Septiembre y octubre de 2014

Fsuc: feromonas en subparcelas sucias. Flim: feromonas en subparcelas limpias. Hlim: hongos en subparcelas limpias. Hsuc: hongos en subparcelas sucias.

Letras diferentes indican valores estadísticamente diferentes.

Figure 5. Weight of Barraganete plantain bunches in $\mathrm{kg}$ per treatments. El Carmen, Manabí, Ecuador. September and October 2014.

Fsuc: pheromones in uncleaned plots. Flim: pheromones in clean plots. Hlim: fungi in cleaned plots. Hsuc: fungi in uncleaned plots.

Different letters indicate statistically different values.

tratamientos con limpieza, tanto con feromonas como con hongos, fue mayor respecto al control. Si se excluyen los controles (Figura 6) el peso del racimo fue superior en las subparcelas con feromonas frente a aquellas en que aplicaron hongos $(\mathrm{F}=9$,243). La longitud de los dedos (Figura 7) presentó diferencias significativas entre los tratamientos de hongos y feromonas $(\mathrm{F}=4,508)$, a favor del tratamiento con feromonas.

La inspección de los cormos tras la cosecha no mostró diferencias significativas entre tratamientos respecto al número de galerías de larvas $(\mathrm{F}=0,782)$.

Se comprobó que la patogenicidad de $B$. bassiana fue mayor sobre $M$. hemipterus que sobre $C$. sordidus (Cuadro 1). Por este motivo está en marcha el aislamiento de poblaciones locales que muestren mayor infectividad sobre C. sordidus.

Las capturas registradas justifican la atención sobre esta plaga. En un estudio similar realizado por Armendáriz

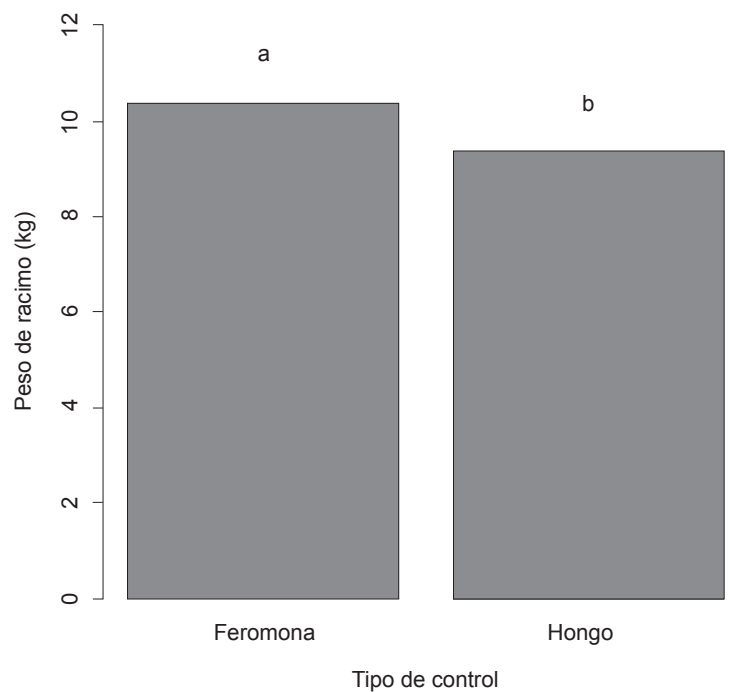

Figura 6. Influencia del tratamiento en el peso del racimo del plátano Barraganete. El Carmen, Manabí, Ecuador. Septiembre y octubre de 2014.

Letras diferentes indican valores estadísticamente diferentes.

Figure 6. Influence of treatment on the weight of Baraganete plantain bunch. El Carmen, Manabí, Ecuador. September and October 2014.

Different letters indicate statistically different values.

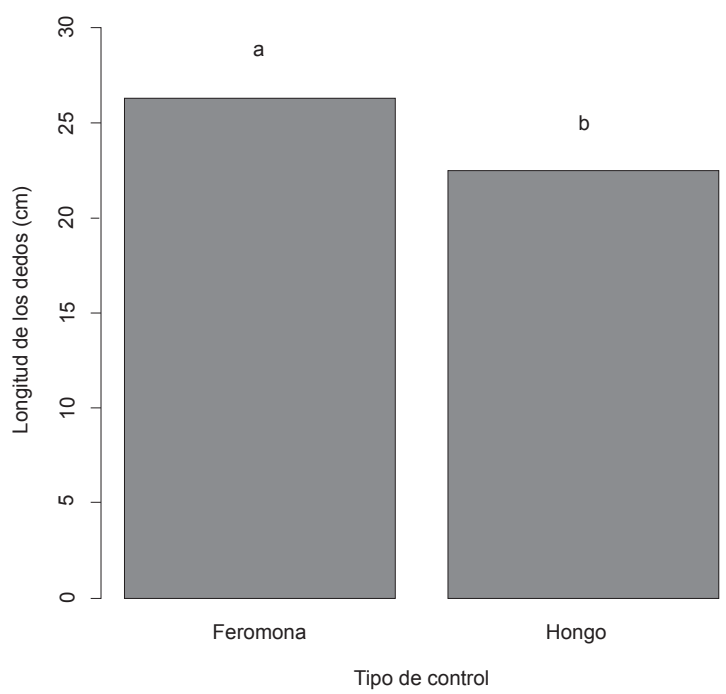

Figura 7. Influencia del tipo de control en la longitud de los dedos de plátano Barraganete. El Carmen, Manabí, Ecuador. Septiembre y octubre de 2014.

Letras diferentes indican valores estadísticamente diferentes.

Figure 7. Influence of the type of control on finger length of Barraganete plantain. El Carmen, Manabí, Ecuador. September and October 2014.

Different letters indicate statistically different values. 
Cuadro 1. Patogenicidad de Beauveria bassiana sobre Metamasius hemipterus y Cosmopolites sordidus. El Carmen, Manabí, Ecuador. Enero- febrero, 2014.

Table 1. Pathogenicity of Beauveria bassiana on Metamasius hemipterus and Cosmopolites sordidus. El Carmen, Manabí, Ecuador. January - February, 2014.

\begin{tabular}{lcccccc}
\hline Infección & Especies & Infectados & $\begin{array}{c}\text { No } \\
\text { infectados }\end{array}$ & $\begin{array}{c}\text { Porcentaje de } \\
\text { infección }\end{array}$ & $\begin{array}{c}\text { Fecha de } \\
\text { infección }\end{array}$ & $\begin{array}{c}\text { Fecha } \\
\text { evaluación }\end{array}$ \\
\hline \multirow{2}{*}{ Primera } & M. hemipterus & 13 & 1 & $92,80 \%$ & $27-01-14$ & $11-02-14$ \\
& C. sordidus & 7 & 8 & $46,70 \%$ & $27-01-14$ & $11-02-14$ \\
\hline \multirow{2}{*}{ Segunda } & M. hemipterus & 11 & 0 & $100 \%$ & $24-02-14$ & $10-03-14$ \\
& C. sordidus & 6 & 31 & $16,20 \%$ & $24-02-14$ & $10-03-14$ \\
\hline
\end{tabular}

et al. (2014) en una platanera cercana de Barraganate de seis años de antiguedad, hubo mayores capturas de picudo, con otro tipo de trampa y otra feromona.

En este estudio, se utilizaron como recipientes botes de plástico de $12 \mathrm{~cm}$ de diámetro superior y 10 $\mathrm{cm}$ de altura y otra feromona comercial sujeta en un alambre en la parte superior del bote, no hubo aplicación de hongos entomopatógenos; la mayor cantidad de adultos capturados (4,29 adultos/trampa/día) se obtuvo a mediados de diciembre de 2013, al igual que en el presente estudio. La falta de medidas de control explica una población superior en la platanera vieja, donde el picudo ha tenido más temporadas para reproducirse, motivado también por la falta de un control del picudo por hongos entomopatógenos. Si la intención del muestreo es fijar umbrales de presencia para decidir una actuación sobre la plantación y tomando el umbral de Muñoz-Ruiz (2007) de diez adultos/trampa/semana, este está superado en al menos ocho de los muestreos (meses de diciembre, marzo, mayo y julio).

El hongo entomopatógeno $B$. bassiana suministrado por el INIAP, esporulado en arroz, ha sido empleado con éxito en la zona de estudio (Armijos, 2008), por lo que aparece como una alternativa válida para el control del picudo (Armendáriz et al., 2014). Las pruebas de laboratorio mostraron que la cepa del hongo es infectiva para el picudo negro.

Los datos finales de cosecha indicaron un efecto positivo significativo de ambos tratamientos en el peso de los racimos frente al control y una discriminación entre subparcelas limpias y sucias.

Si los tratamientos disminuyen la presión del picudo, las plantas deberían presentar un mejor desarrollo y cosechas más altas, teniendo en cuenta los hábitos y los daños infligidos por la plaga (Gold y Messiaen, 2000), en especial la restricción de movimientos de sabia provocada por las galerías del picudo. Paradójicamente a la hora de estimar la presencia y actividad de larvas en el cormo tras la cosecha, no se encontraron diferencias significativas entre tratamientos. Un $38,67 \%$ de los cormos presentaban galerías de larvas, con una media de 0,83 galería/planta en el total de las sesenta plantas inspeccionadas. Ello pudo deberse a la presencia del inóculo inicial en la plantación, asociado a una deficiente desinfección del material de siembra, a pesar del uso de un tratamiento químico con clorotalonil. Los datos de un segundo ciclo de cultivo podrián arrojar diferencias significativas si efectivamente los tratamientos de hongos y feromonas tuvieran un efecto de protección respecto a la oviposición de los adultos.

Se han enunciado varios aspectos que se deben contemplar para el control del picudo los cuales son: elección de variedades, buscando las más tolerantes; cuidado con el material de siembra, limpieza y desinfección; estimación de la población del picudo mediante capturas regulares; captura masiva con feromonas o con otro tipo de trampas; aplicación de hongos entomopatógenos; limpieza de las parcelas, eliminando los restos de poda y plantas caídas; cuidado cultural de la platanera; deshierbe, deshije, desoje, abonado y riego para fomentar el vigor de la planta y por último, renovación de plantaciones en caso de altos ataques (Armendáriz et al., 2014).

Los resultados de este estudio no mostraron mayores capturas de adultos con trampas de feromonas en las subparcelas libres de residuos. Ello pudo estar relacionado con el continuo aporte de materia orgánica en los procesos de deshierbes, deshijes y deshojes que sucesivamente han acercado las condiciones de las subparcelas limpias y sucias, a pesar de algunas limpiezas parciales sucesivas. 
Respecto a los parámetros agronómicos (altura de plantas y perímetro del pseudotallo a $30 \mathrm{~cm}$ ), Gold et al. (2004) en un ensayo en banano en Uganda, no encontraron diferencias en el perímetro y altura de plantas en parcelas infestadas y libres durante los tres primeros años. Sin embargo, en este estudio sí se obtuvieron diferencias significativas, indicando que los tratamientos ya han tenido un efecto positivo sobre la plantación en su primer año.

Las capturas con feromonas se mostraron más efectivas que las capturas con pseudotallos (De Graaf et al., 2005); trampa utilizada en este estudio en las subparcelas con hongos. Sin embargo, las capturas de adultos pueden no ser suficientes para la protección del cultivo, como lo indicaron Rhino et al. (2010). Se necesitan otros métodos de control, incluidos los culturales (Armendáriz et al., 2014), entre ellos el de la limpieza del material de siembra, con productos químicos o calor, aparece como determinante, ya que con ello se elimina la población inicial y se retardan los daños producidos por infecciones posteriores en la parcela.

Las capturas realizadas en el presente trabajo se mantuvieron a lo largo de los once meses de estudio, indicando la gravedad del problema, que afecta a otras zonas de producción.

\section{AGRADECIMIENTOS}

Este trabajo no hubiera podido ser realizado sin el apoyo de la SENESCYT, que con su programa PROMETEO ha posibilitado las investigaciones de Santiago Ulloa e Ignacio Armendáriz. De la misma forma, la Universidad de las Fuerzas Armadas ESPE ha brindado sus infraestructuras para la realización de distintos ensayos. Gracias igualmente a la familia Ulloa de El Carmen, por brindar desinteresadamente su finca y a Bismarck Guerrero por la toma laboriosa de datos y su curiosidad que en ocasiones nos ayudó a dilucidar extremos confusos, una mención especial para la Dra. María Ángeles Padilla por sus consejos y a ScyllAgro por la provisión de material de investigación. Finalmente, un agradecimiento a FENAPROPE por su apoyo en la difusión de conocimientos.

\section{LITERATURA CITADA}

Abera-Kalibata, A.M., A. Hasyim, C.S. Gold, and R. Van Driesche. 2006. Field surveys in Indonesia for natural enemies of the banana weevil, Cosmopolites sordidus (Germar). Biol. Control 37:16-24.

Aguilar, C.H., F. Lasalita-Zapico, J. Namocatcat, A. Fortich and R.M. Bojadores. 2014. Farmers' perceptions about banana insect pests and integrated pest management (IPM) systems in SocSarGen, Mindanao, Philippines. IPCBEE 63(5):22-27.

Akello, J., T. Dubois, D. Coyne, and S. Kyanamywa. 2008 Endophytic Beauveria bassiana in banana (Musa spp.) reduces banana weevil (Cosmopolites sordidus) fitness and damage. Crop Protection 27:1437-1441.

Armendáriz, I., P.A. Landázuri, y S. Ulloa. 2014. Buenas prácticas para el control del picudo del plátano en Ecuador. ESPE. http://giat.espe.edu.ec/wp-content/ uploads/2014/06/Picudo-del-pl\%C3\%A1tano2014. pdf. (consultado 12 dic. 2014).

Armijos, F. 2008. Principales tecnologías generadas para el manejo del cultivo de banano, plátano y otras musáceas. Programa Nacional de Banano, Plátano y otras Musáceas. Boletín Técnico no. 131. INIAP, Guayaquil, ECU.

De Graaf, J.P., A.T.S. Govender, Schoeman, and A. Viljoen. 2005. Efficacy of pseudostem and pheromone seasonal trapping of the banana weevil Cosmopolites sordidus in South Africa. Int. J. Pest. Manag. 51:209-218.

Fancelli, M., A.B. Dias, J.I. Delalibera, S. Cerqueira de Jesus, A. Souza do Nascimento, S. De Oliveira e Silva, R. Correa Caldas, and C.A. da Silva Ledo. 2013. Beauveria bassiana strains for biological control of Cosmopolites sordidus (Germ.) (Coleoptera: Curculionidae) in plantain. BioMed Res. Int. 2013: 184756. doi: $10.1155 / 2013 / 184756$

Fundación PRODUCE de Guerrero, A.C. 2012. Agenda de innovación del plátano. Fundación PRODUCE de Guerrero A.C, MEX.

Gold, C.S., G.H. Kagexi, G. Night, and P.E. Ragama. 2004. The effects of banana weevil, Cosmopolites sordidus, damage on highland banana growth, yield and stand duration in Uganda. Ann. appl. Biul. 145:263-269.

Gold, C., y S. Messiaen. 2000. El picudo negro del banano: Cosmopolites sordidus. Plagas de Musa. 
Hoja divulgativa No. 4. https://www.google.com/ search? $\mathrm{q}=\mathrm{www}$.bioversity $\% 2$ Fintenational.org $\% 2 \mathrm{Ff}$ ileadmin $\% 2$ Fbioversity $\% 2$ Fpublications $\% 2 \mathrm{Fpdfs} \%$ 2F696_ES.pdf\&oq=www.bioversity $\% 2$ Fintenational. org\%2Ffileadmin $\% 2$ Fbioversity $\% 2$ Fpublications $\% 2 \mathrm{~F}$ pdfs\%2F696_ES.pdf\&aqs=chrome..69i57j69i58.999j $0 \mathrm{j} 4 \&$ sourceid $=$ chrome $\& \mathrm{ie}=\mathrm{UTF}-8 \# \mathrm{q}=\mathrm{E} 1+\mathrm{Picudo}+\mathrm{Ne}$ gro+del+Banano:+Cosmopolites+sordidus.+Plagas $+\mathrm{d}$ e+Musa.+Hoja+Divulgativa+No.+4.+ (consultado 12 dic. 2014).

Gold, C.S., J.E. Pena, and E.B. Karamura. 2001. Biology and integrated pest management for the banana weevil Cosmopolites sordidus (Coleoptera: Curculionidae). Integrated Pest. Manag. Rev. 6:79-155.

Gold, C.S., P.E. Ragama, R. Coe, and N.D.T.M. Rukazambuga. 2005. Selection of assessment methods for evaluating banana weevil Cosmopolites sordidus (Coleoptera: Curculionidae) damage on highland cooking banana (Musa spp., genome group AAA-EA). B. Entomol. Res. 95:115-123.

INEC (Instituto Nacional de Estadística y Censo). 2011. Datos estadísticos agropecuarios. http://www. ecuadorencifras.gob.ec/wp-content/descargas/ Presentaciones/PRESENTACION_Espac.pdf (consultado 31 mar. 2015).

INIBAP (International Network for the Improvement of Banano and Plantain). 1998. Producción del banano orgánico y/o ambientalmente amigable. En: F.E. Rosales, et al., editores, Memorias del taller internacional realizado en la EARTH 27-29 de julio de 1998. Centro Editorial Srl, San Pedro Sula, HON. p. 1-264.

Jiménez, J.A. J.C. López, y A. Soto. 2012. Patogenicidad de dos nematodos entomopatógenos sobre Metamasius hemipterus sericeus (Coleoptera: Curculionidae). Bol. Cient. Mus. Hist. Nat. Univ. Caldas 16(2):87-97.
Lopes, R.B., Laumann, R.A., Moore, D., Oliveira, M.W.M. and Faria, M. 2014. Combination of the fungus Beauveria bassiana and pheromone in an attract-andkill strategy against the banana weevil, Cosmopolites sordidus. Entomologia Experimentalis et Applicata 151:75-85. doi: 10.1111/eea.12171

MAGAP (Ministerio de Agricultura, Ganadería, Acuacultura y Pesca). 2015. Provincia de Manabí-Mapa de suelos (variable taxonomía). http://geoportal.agricultura.gob. ec/mapas/manabi/mapa_suelos_taxonomia_manabi. pdf (consultado 9 jul. 2015).

Masanza, M., C.S. Gold, and A. Van Huis. 2005. Distribution, timing of attack, and oviposition of the banana weevil, Cosmopolites sordidus, on banana crop residues in Uganda. Entomol. Exp. Appl. 117:119-126.

Muñoz-Ruiz, C. 2007. Fluctuación poblacional del picudo negro (Cosmopolites sordidus Germar) del plátano (Musa AAB) en San Carlos, Costa Rica. Tecnología en Marcha 20:24-41.

Quijije, R. 2003. Desarrollo de tecnologías limpias para el manejo del picudo negro (Cosmopolites sordidus), en plátano. Tesis, Universidad de Guayaquil, ECU.

Rhino, B., M. Dorel, P. Tixier, and J.M. Rise. 2010. Effect of fallows on population dynamics of Cosmopolites sordidus: toward integrated management of banana fields with pheromone mass trapping. Agric. For. Entomol. 12:195-202.

ScyllAgro. 2015. Fiche des données de sécurité. http:// coopagri-noumea-nc.net/pdfcoop/charencon $\% 20$ du\%20bananier\%20nc.pdf (consultado 4 jul. 2015).

Shapiro, S., and M.B. Wilk. 1965. An analysis of variance test for normality (complete samples). Biometrika 52: 591-611.

Traore, L., C.S. Gold, J.G. Pilon, and G. Boivin. 1993. Effects of temperature on embryonic development of banana weevil, Cosmopolites sordidus Germar. Afr. Crop Sci. J. 1:111-116. 
